\section{Delay of punishment in shock-elicited aggression}

\author{
C. D. WETZEL
}

San Jose State College, San Jose, Calif. 95114*

Forty restrained rats, given tailshocks to elicit biting on a target, were given a second shock contingent on the biting at different delay times. Comparisons made in different phases among four $(0,3,6$, and $9 \mathrm{sec})$ and two $(0$ and $9 \mathrm{sec})$ conditions showed longer delays of punishment to produce less suppression of the biting, though not significantly so in the former comparison. The results indicated that shock stimuli in elicited aggression have dual functional properties where longer delayed and free shocks serve to elicit, while more immediately contingent shocks serve to suppress biting.

Numerous experiments have demonstrated the reflexive-like aggression elicited in animals given aversive stimulation. Ulrich \& Azrin (1962) defined aggression in terms of the raised stereotyped fighting repeatedly elicited between paired rats given footshocks. Defining aggression in terms of attacks on inanimate objects, Azrin, Rubin, \& Hutchinson (1968) have also shown solitary rats to bite on metal and wood targets following aversive tail shocks. An interesting outcome of this line of investigation has been the more recent finding that a shock used to elicit aggressive behavior can also be used to suppress that same behavior. Using restrained squirrel monkeys, both Ulrich, Wolfe, \& Dulaney (1969) and Azrin (1970) elicited a high level of biting on a rubber-hose target with noncontingent (NC) fixed-interval tail shocks. In later sessions, the addition of a second shock presented immediately contingent on the elicited bites served to suppress the biting to a low level. The Azrin investigation further demonstrated the suppression to be an increasing function of the punishing shock's intensity.

The finding that a shock stimulus could serve as both a punisher and elicitor raises an interesting question when combined with the result of another punishment study. Camp, Raymond, \& Church (1967) showed that increasing delays in the delivery of a punishment lead to progressively less suppression of a rat's barpressing. Furthermore, the longer the punisher was delayed, the more its effects were like the relatively nonsuppressive effects of noncontingent shock. It is possible that a similar delay effect would result in the elicited aggression situation. Thus, biting in response to NC shocks would be expected to be relatively suppressed to more immediate delayed punishments. Longer delays would be expected not

* Now at the University of California. Riverside, Calif. 92502 to suppress, and in fact might possibly facilitate, biting, the effects of longer delays being more like those of the noncontingent shocks that originally served to elicit biting. The purpose of the present experiment, then, was to test the prediction that more biting would accompany longer temporal delays in the delivery of punishment. SUBJECTS AND APPARATUS

Forty Long-Evans male hooded rats were maintained in separate cages and allowed free access to food and water. All Ss were approximately 110 days of age on the first day of introduction into the apparatus.

The biting apparatus followed the design of Azrin et al (1968) and consisted of three components: a biting target, a Plexiglas restraining tube, and a tail-restraining rod. Briefly, the biter consisted of two 1-in.-wide 1/16-in.-thick sheets of stainless steel separated by a $3 / 64$-in. air space at the 1-in. length of the plates projecting to the animal. When the two plates of steel were squeezed together by a bite, a microswitch contact between the plates closed to record the bite on a recorder. The Plexiglas restraining tube was $10^{1 / 2}$ in. long, with a $21 / 2$-in. diam, and fitted with brass rods to prevent the animal from twisting or backing out of the tube. The animal's tail was secured to a wooden rod with strips of surgical tape. Two $1 / 2-$ in.-wide stainless steel electrodes, $1 \frac{1 / 2}{2}$ in. apart, with the the animal's tail, were held in place on the tail by springs. Electrode paste was used to insure proper contact, and an Applegate constant-current stimulator, Model 250, served as the shock source. PROCEDURE

After 2 days of 5-7 min of handling, the Ss were assigned randomly to one of the four groups (10 Ss each) and put through four phases lasting two sessions each. Each of the eight daily sessions of restraint in the apparatus lasted $30 \mathrm{~min}$.

During the first phase of training, the animals were simply restrained in the apparatus so as to provide a nearest being $2^{1 / 2}$ in. from the base of no-shock baseline of biting. The biting target was positioned for each $S$ approximately $1 / 2$ in. in front of the S's nose. Ink marks corresponding to marks on the tail-restraining rod were made on each S's tail to facilitate similar repositioning of the animals in the subsequent sessions.

The second phase involved the noncontingent delivery of shock to all four groups of animals for two sessions. During this phase, 60 shocks per session were delivered to the animal's tail at fixed intervals of 30 sec. These NC 30 shocks were continued through the remaining experimental phases. The shock was $200 \mathrm{msec}$ in duration, and the intensity was $5 \mathrm{~mA}$, as measured on the shock-source meter with the tail electrodes crossed. This procedure yielded a rough baseline of shock-elicited biting against which the later effects of punishment could be compared.

The third phase of training involved the punishment of biting elicited by the noncontingent shocks. Scheduling equipment was programmed such that the first bite after an NC 30 shock delivered an identical second shock to the animal. This response-contingent or punishing shock was presented to the four experimental groups, each at a different temporal delay after the first elicited bite:" $0,3,6$, or 9 sec. If no bite occurred in the interval between successive NC 30 shocks, the response-contingent shock was not received in that interval.

The fourth phase of training shifted the four groups to different temporal delay values. The shift was an attempt to demonstrate the power of the independent variable in manipulating the biting behavior and also to see if the groups could be further separated by using only two groups. The 0 - and 3 -sec delay groups in the previous third phase were now both shifted to a 9-sec delay of punishment in the fourth phase. Likewise, the 6- and 9 -sec delay groups were shifted to a 0 -sec delay of punishment.

\section{RESULTS}

Figure 1 shows the mean performance of the four groups over Shock Sessions 3-8. As a measure of reactivity to the NC 30 shocks, the groups were compared on the number of 30-sec shock intervals in which any biting occurred. The measure consequently had an upper limit of 60 -the number of NC 30 shocks delivered in a given session. An alternative measure, biting frequency, proved to be an unstable measure, allowing considerable variation.

As in the similar Azrin et al (1968) demonstration of the pain-aggression relationship, an abrupt increase of biting on the target followed the introduction of aversive stimulation. 


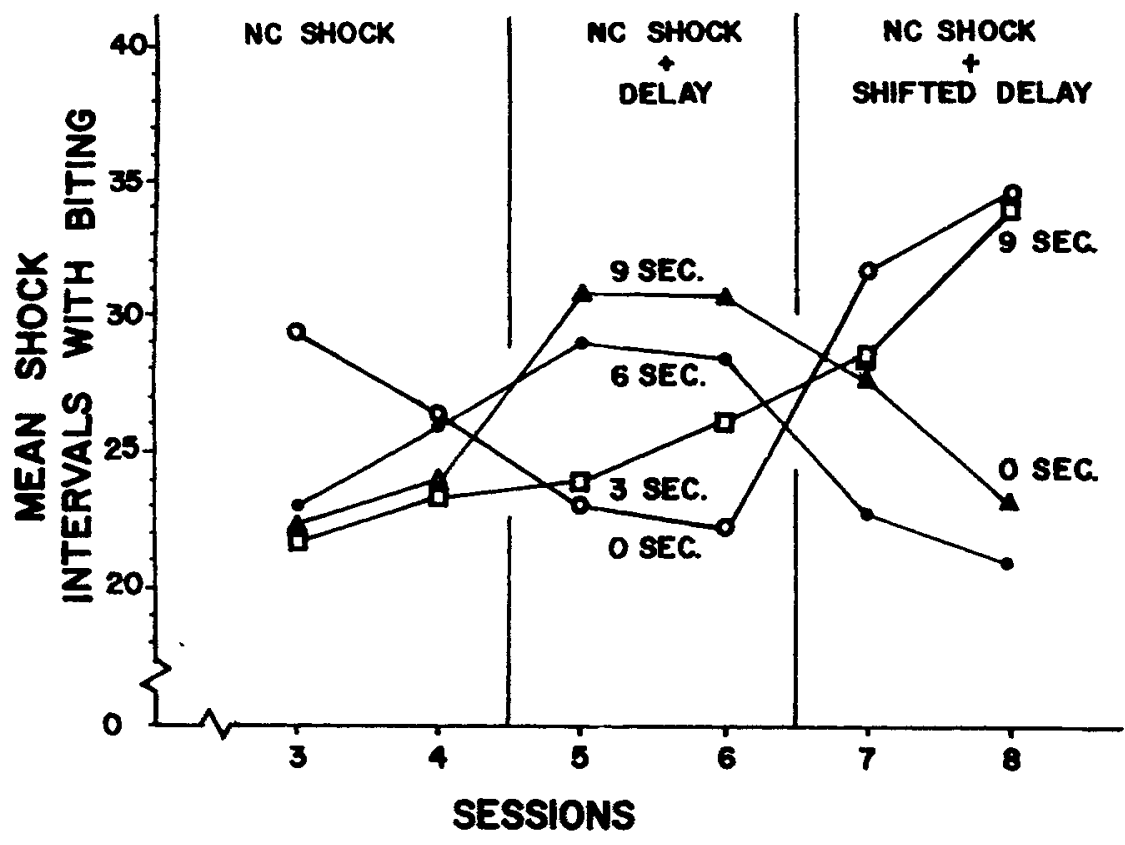

Fig. 1. Group means for the number of 30-sec shock intervals in which any biting occurred. Accompanying numbers are delay of punishment times.

The mean frequency of biting for all Ss during the first two no-shock sessions was 14 , while the corresponding figure for the NC 30 Shock Sessions 3 and 4 was 128 (not shown).

In the figure, it can be seen that the addition of the different delayed punishments in Sessions 5 and 6 acted to spread the four groups upward slightly on the number of shock intervals measure. The experimental procedure of punishing only the first of any bites during an NC 30 shock interval led to a relative effect, where the suppression of the 0 -sec delay group's biting was not complete. The groups were distributed in the hypothesized rank order, with more biting accompanying longer delays of punishment. The difference among the groups at Session 6 , however, was not significant $[F(3,36)=.67, p>.05]$.

In Session 8 during the final phase of the experiment, the two 9-sec delay groups had a combined mean 34.3 number of shock intervals with biting, while the two 0 -sec delay groups had a mean of 22.1. It is clear from the figure that the shift in delay times from the previous experimental phase produced a corresponding change in the direction of the groups' performance: immediately contingent shock served to suppress biting, while the longer delayed shock served to elicit or further facilitate biting. The separation of the groups was statistically significant $[F(1,38)=4.18, p<.05]$.

DISCUSSION

The hypothesis predicting more biting with longer temporal delays in the delivery of punishment was supported in the latter phase of the experiment. The finding of a delay of punishment effect for shock-elicited aggression is in agreement with the analogous delay effect shown for barpressing (Camp et al, 1967). Such an effect, however, was not statistically demonstrated among the four delay groups, though the groups were ranked with a positive association between biting and delay times. In view of the effect shown in the two-group comparison, a probable explanation for the four-group outcome is that the 3 -sec intervals between the group delay times were not large enough for a separation of the groups.

In addition to showing a delay-of-punishment effect, the results were also indicative of the dual functionality of aversive stimuli in shock-elicited behavior. As with other studies (Azrin, 1970; Ulrich et al, 1969), shock stimuli differing only in their temporal relation to the biting were used to both elicit and suppress the behavior.

With longer delayed shocks increasing the level of biting, the question arises as to whether the biting was further facilitated over the base level expected to only the on-going NC 30 shocks. Indeed, the figure shows that both the 9 -sec conditions exceeded the level of biting elicited in the earlier sessions. Since fighting frequency has been shown to be related to shock frequency (Ulrich \& Azrin, 1962), it would seem likely that the trend of both more frequent shocks and biting in the longer delayed conditions would indicate a facilitation of biting (note that the biting measure is also equivalent to the number of punishments delivered). Without an equally experienced NC 30 group for comparison, however, such a conclusion is confounded with the tendency for elicited aggression to increase with successive shock sessions (Creer \& Powell, 1971; Powell \& Creer, 1969).

The dual properties of elicitation and suppression shown by the aversive stimuli in the present study point to the overlapping place of the biting response with respect to operant-respondent categories. While the biting occurred to the stimulus presentations without any prior conditioning, it was, however, a result of only $40 \%$ of the total prepunishment shocks presented (i.e. NC 30 shocks in Sessions 3 and 4). This level of biting was similar to that previously found on a metal target (Azrin et al, 1968). Observation of the series of movements involved in the biting attacks indicated that the shock-bite relation was not always a discrete one-to-one relationship. Biting attacks appeared, rather, to be only one element of a molar reaction which itself involved vocalizations, struggling, and escape behaviors. Thus, while the biting response shows a similarity to respondent behavior in terms of the eliciting operations involved, it evidences a considerable operant component witnessed in its susceptibility to consequential operations, its composite nature, and its mediocre probability of occurrence to its eliciting stimulus. The present discussion is in accord with the previous conclusion (Powell \& Creer, 1969) that the shock-aggression relationship is more than simple and possesses both respondent and operant characteristics.

\section{REFERENCES}

AZRIN, N. H. Punishment of elicited aggression. Journal of the Experimental Analysis of Behavior, 1970, 14, 7-10.

AZRIN, N. H., RUBIN, H. B., \& HUTCHINSON, R. R. Biting attack by rats in response to aversive shock. Journal of the Experimental Analysis of Behavior. $1968,11,633-639$.

CAMP, D. S., RAYMOND, G. A., \& CHURCH, R. M. Temporal relationship between response and punishment. Journal of Experimental Psychology, $1967,74,11$ 4-123.

CREER, T. L., \& POWELL, D. A. Effect of repeated shock presentations and different stimulus intensities on shock-induced aggression. Psychonomic Science, 1971, 24, 133-134.

POWELL, D. A., \& CREER, T. L Interaction of developmental and environmental variables in shock elicited aggression. Journal of Comparative \& Physiological Psychology, 1969, 69 219-225. 
ULRICH, R. E., \& AZRIN, N. H. Reflexive fighting in response to aversive stimulation. Joumal of the Experimental
Analysis of Behavior, 1962, 5, $511-520$. ULRICH, R. E., WOLFE, M., \& DULANEY, S. Punishment of shock-induced aggression. Journal of the Experimental Analysis of Behavior, 1969,12 , 1009-1015. 